\title{
Implementation of a Projection \\ Onto Convex Sets Iteration Based \\ Image Coder
}

\author{
by \\ M. Reha 'Civanlar \\ Sarah A. Rajala \\ and \\ Thomas K. Miller
}

Center for Communications and Signal Processing

Department of Electrical and Computer Engineering

North Carolina State University

August 1986

CCSP-TR-86/20 


\section{Abstract}

Projection onto convex sets iteration based image coding, where efficiently encodable sets are used to describe an image, is a recent approach to image compression. The technique allows the use of a variety of sets to encode an image. The focus of this paper, however, will be on two particular sets: the set of images whose cosine transform is known for certain frequencies and the set of images which are nonzero over a specified region. These sets can be used to encode interframe difference pictures of video teleconferencing images. A drawback of this new type of codec is its computational complexity. In this paper, an architecture to overcome this drawback as well as other implementation related issues such as the quantization effects will be discussed.

\section{Introduction}

The projection onto convex sets (POCS) iteration based image coding techniques may be considered as a generalization of transform coding where each image is described by the set of its projections onto the selected transform's basis. If the basis frames are denoted by $b_{i}(m, n) i=1, \ldots, k$, the original image, $\mathrm{f}(\mathrm{m}, \mathrm{n})$, can be written as

$$
f(m, n)=\sum_{i=1}^{i=k} \alpha_{i} b_{i}(m, n)
$$

where, $\alpha_{i}$ coefficients are used to encode the image. In general, to encode an $N \times N$ image, $N \times N \quad \alpha$ coefficients are required. However, by using the correlation structure of a group of images, the basis can be selected so that each image may be described by much fewer coefficients. The Karhunen-Loeve expansion is the well known technique to obtain such a basis for a group of images with a known correlation structure. A performance 
very close to that of the Karhunen-Loeve expansion can be obtained by using twodimensional (2D) Fourier or cosine transforms. Since these transforms use standard basis images and their computational complexity is much lower than that of the KarhunenLoeve, the cosine transform is widely used in practical image coding. ${ }^{1}$

An alternative approach to image coding is to transmit parameters describing convex sets which are known to contain the image to be encoded. The image is a member of the intersection of these sets and if the intersection set is small enough, any member of it will be close to the encoded image. A member of the intersection set can be found using the POCS method which is based on a technique for finding common points of closed and convex sets in a Hilbert space. ${ }^{2}$ This method has been successfully applied to various signal processing problems including: bandlimited signal extrapolation, tomographic reconstruction, and signal restoration. ${ }^{3}$ The main result from the POCS theory can be stated as Let $C_{0}=\cap C_{i}$ be nonempty, where each $C_{i}$ is a closed and convex set in a finite dimensional Hilbert space. Let $P_{i}[$.$] be the projection operator for the set$ $C_{i}$, which is determined by solving the constrained minimization problem

$$
\begin{gathered}
\min \left\|f-f^{0}\right\|^{2} \\
f \in C_{i}
\end{gathered}
$$

where, $f^{0}$ is the vector to be projected. Then the iteration given by

$$
f^{k+1}=P_{1} P_{2} \ldots P_{M}\left[f^{k}\right]
$$

converges to a point in $C_{0}$.

The POCS based coding includes the transform coding as a special case since, each coefficient of a transform coder defines a hyperplane which contains the encoded image. 
The mathematical description of these $N \times N$ dimensional hyperplanes can be written as

$$
H_{i}=\left\{f\left|<b_{i}, f\right\rangle=\alpha_{i}\right\}
$$

where, $\langle.,$.$\rangle is the inner product and these hyperplanes are nothing but convex sets. To$ model the quantization of the transform coefficients, eq. (4) can be modified as

$$
H_{i q}=\left\{f \mid \alpha_{i}-\Delta \leq\left\langle b_{i}, f\right\rangle \leq \alpha_{i}+\Delta\right\}
$$

where, $\Delta$ is the quantization error. $H_{i q}$ is also a convex set of images containing the encoded image.

In the case of two convex sets with an empty intersection, the POCS method can be used to find the closest point of one of the sets to the other one. A formal statement of this result, which has been proven in ${ }^{4}$ and used in ${ }^{5}$ to design windows for power spectrum estimation, is as follows

Let $C_{1}$ and $C_{2}$ be two disjoint closed convex sets in a Hilbert space and $C_{1}$ is bounded. Then, there exist an $f \in C_{1}$ whose distance to $C_{2}$ is minimum, and $f$ is such an element iff it is a fixed point of the operator $P_{1} P_{2}[$.$] , where, P_{i}$ is the projection operator onto $C_{i}$.

The use of this result in POCS based image coding is presented by Santago. ${ }^{6,7}$ In this paper, the implementation related issues of this application will be discussed.

\section{Interframe difference picture encoder}

At this point, there is no methodology to define the optimal collection of convex sets for a certain image coding application. A group of sets which can be used with most of the image sequences is discussed in. ${ }^{8}$ It may be possible to develop a generic architecture to implement the POCS based coders, however, in order to design a practical codec the 
convex sets to be employed must be well defined. One approach to determine the sets is to use the prominent characteristics of a group of images to be encoded. Such an approach can be taken in encoding the interframe difference pictures of the video teleconferencing image sequences because these images contain large, zero intensity regions.

The first technique exploiting the sparsity of the interframe difference pictures is the conditional replenishment, ${ }^{9}$ where the addresses and the magnitudes of non-zero pixels are transmitted. A number of variations of this approach have been proposed in the literature. The fundamental problem with all of these has been the variable data rate caused by the varying amount of motion. A solution for this problem may be through the use of motion compensation which enlarges the zero intensity regions through the estimation of the motion of the objects in the picture sequence. Cases where the next frame is almost completely estimated from the previous frames using the motion compensation are reported in literature. ${ }^{10}$ However, such algorithms are complicated and although the number of the remaining non-zero pixels is small, it still varies from frame to frame.

The use of interframe difference pictures in transform coding is well established. ${ }^{11}$ Transform coding the interframe difference pictures is equivalent to using a simple DPCM in time domain where the set of transform coefficients of each frame is used as an estimate for that of the next frame. Because of the interframe correlation such an approach reduces the dynamic range of the transform coefficients, and in turn, reduces the quantization error. The POCS iteration based coder combines the conditional replenishment technique, which is a space domain method, with the hybrid transform/DPCM coding. This is accomplished using two convex sets. The first set consists of images which can be 
described by a certain number of cosine transform coefficients and the second set is a set of images whose intensities are specified only in a certain region called "mask".

The POCS technique can be used in two different ways to encode the interframe difference pictures based on these sets. The first approach is to transmit the selected transform coefficients and the mask to the receiver. In this case, the receiver looks for an image in the intersection of the sets $C_{F}$ and $C_{S}$ given by

$$
\begin{gathered}
C_{F}=\left\{f \mid b_{1} \leq S[C[f]] \leq b_{2}\right\} \\
C_{S}=\{f \mid \bar{M}[f]=0\}
\end{gathered}
$$

where, the operator $\mathrm{C}[$.$] is the cosine transform, the operator \mathrm{S}[$.$] is a coefficient selector,$ $b_{1}$ and $b_{2}$ are vectors containing the lower and upper bounds for the selected transform coefficients derived from the quantized coefficients as in eq. (5), and $\bar{M}$.] is an operator selecting the pixels outside the mask. The projection of an image onto the set $C_{F}$ can easily be obtained by changing the cosine transform coefficients, which are outside of the bounds, to the closest bound. The projection onto $C_{S}$ is accomplished by assigning zero intensities to the outside of the mask pixels. In this setting, the receiver seems to be the only place which uses the iterations, however, since the decoded image must be known by the transmitter, the same iteration must also be performed at the transmitter. The second approach, eliminates the iterations at the receiver by using the non-intersecting sets version of the POCS theory. In this technique, the definition of the sets are slightly modified and given by

$$
C_{F}=\{f \mid \bar{S}[C[f]]=0\}
$$




$$
C_{S}=\{f \mid M[f]=c\}
$$

where, the operators $\bar{S}[$.$] and M[$.$] are the complements of the operators S[.] and \bar{M}[$.$] of$ eq. (6) and the vector c contains the intensities of the pixels inside the mask. If an image in the intersection of $C_{F}$ and $C_{S}$ can be found, it can be encoded exactly using only the selected cosine transform coefficients. On the other hand, if the intersection is empty, the POCS method will find the closest image, described by the selected number of coefficients, to the image to be encoded considering only the pixels inside the mask. It should be noted that, the pixels outside the mask are treated as don't cares in this approach and they are modified to obtain an image which is suitable to encode with the limited number of transform coefficients. Given the transform coefficients and the mask, the operations to be performed at the receiver are the inversion of the received set of transform coefficients and assigning zero intensities to the outside of the mask pixels. Because of the reduced hardware requirements, this approach has been selected for the implementation.

The advantage of the new method over the space domain techniques is the reduced variation in the data rate. In the case of conditional replenishment, the amount of data to be transmitted is proportional to the number of non-zero pixels. In the POCS based coder, the number of transform coefficients to be transmitted can be fixed without a noticeable effect on the quality of the picture. A secondary advantage of the new approach follows from its transform domain base: the quantization errors are not localized.

If the bandwidth used to transmit the mask is ignored, the new codec is always better than the classical transform coder. This is because of its flexibility in modifying the 
outside of the mask pixels of the image so that the optimal image to be encoded with the given set of transform coefficients can be obtained. In order to use the same bandwidth with a classical transform coder, the extra bandwidth used to encode the mask must be deducted from the bandwidth allocated to the transform coefficients. There is no formal proof for the superiority of the new codec over a transform coder considering the additional bandwidth required by the mask, however, the simulation results reported in ${ }^{6,7,12}$ demonstrated that, using the new method, considerable improvement can be obtained in most of the cases.

\section{Implementation related issues}

\section{Encoding the mask}

The mask is a binary picture indicating the locations of the pixels whose values have changed with respect to the previous encoded frame. In a typical teleconferencing sequence, the percentage of the pixels whose values have changed is less than $20 \%$ for about $90 \%$ of the frames. ${ }^{13}$ In addition to the sparsity of the mask, the changes occur in clusters around the edges of the original picture. This property of the mask pictures is used in designing codecs based on conditional replenishment to transmit the addresses of the changes as clusters using run-length codes. ${ }^{13}$ However, the codes designed to transmit clusters are very inefficient in encoding isolated pixels. In, ${ }^{13}$ the effect of this is reduced by removing the isolated pixels indicating change, and bridging the small gaps between the clusters. 
Since it is very unlikely to have motion confined to a single pixel, most of the isolated changes are because of noise and elimination of them will not degrade the picture quality. Based on this observation, the raw mask picture, which is generated by thresholding the interframe difference picture, is scanned to remove the isolated non-zero pixels.

After the removal of the isolated pixels, the sizes of the clusters can be enlarged to include the pixels between the neighboring clusters. This reduces the number of clusters increasing the coding efficiency. The superiority of the new codec to a conditional replenishment approach is its flexibility in the modification of the mask. In the conditional replenishment approach, each pixel, which is artificially included in the mask, must be quantized. That is, enlarging and bridging the clusters will increase the efficiency of the mask coder, however, the quantization of the modified pixel values will reduce the affect of this improvement. In the POCS based codec, the effect of the mask size on the required number of transform coefficients is not very important. This makes it possible to use a fixed rate coder for the mask.

\section{Quantization of the coefficients}

Bit allocation for the quantization of the cosine transform coefficients is studied by many researchers. In ${ }^{14}$, a visually weighted quantization scheme for encoding still frames using as low as 0.5 bits per pixel (bpp) is presented. Since, in this application the interframe difference pictures are to be encoded, more compression can be obtained by using fewer coefficients and quantization levels. In the simulation studies, reasonable images can be obtained using $0.1 \mathrm{bpp}$ for the transform coefficients. 


\section{Number of iterations}

The exact number of POCS iterations required for the convergence is unknown. Since these iterations are the most computationally intensive part of the codec, a varying number of iterations cannot be afforded. Furthermore, the speed of convergence of the POCS technique is much faster during the initial iterations. Because of these reasons and the results obtained in the simulation studies, the number of iterations is fixed to five.

\section{Proposed architecture}

The POCS coder requires a substantial number of computations per frame. Fixing the number of POCS iterations at five requires 10 discrete cosine transform (DCT) computations per block per frame. In addition, the binary mask must be generated before the POCS iterations begin. Since the generation of the difference picture is recursive, all of these computations must be completed in one frame time. This section analyzes these constraints for the proposed POCS transmitter architecture presented in figure 1.

Figure 1 - POCS transmitter.

Facsimile coding techniques may be used to encode the mask exactly, ${ }^{15}$ but these techniques generate variable length codes (facsimile transmission is not subject to realtime constraints). In order to achieve a fixed length mask encoding, the mask derived directly from the difference picture is modified so that it can be represented by a run length code whose size is determined by a priori statistical analysis. The algorithm chosen for this modification is as follows: First, remove all isolated pixels by complementing all "1" mask pixels which are completely surrounded by "0" pixels, and vice-versa. Next, 
compute a histogram of the run lengths. From this histogram the number of runs which must be eliminated to achieve the fixed length code is determined. Finally, the required number of the shortest runs are removed, generating a suitably encodable mask. This algorithm has the desirable properties that the computation time for the mask modification is constant, and that the noise introduced is distributed evenly over the mask. The time required to compute the modified mask for an $N \times N$ image processed in raster scan order is $N \times N+2 \times N+2$, divided by the data rate.

Recently, a VLSI chip capable of computing an 8 point DCT at a sustained rate of 800 ns per 8 point DCT has been reported..$^{16}$ An array of sixteen of these chips is thus capable of computing an $8 \times 8$ DCT with the same throughput. Since the forward and inverse DCT's use the same kernel, the inverse DCT can be computed at the same rate using another array of sixteen chips with a slightly modified architecture. For an image of size $256 \times 256$, the $10,2408 \times 8$ DCT computations required for five POCS iterations would require $4.10 \mathrm{~ms}$. Assuming that the mask processing elements are clocked at the same rate as the DCT array $(10 \mathrm{MHz})$, the time required to compute the modified mask is $6.61 \mathrm{~ms}$. The total computation time around the feedback path is $10.71 \mathrm{~ms}$, well within the $33.33 \mathrm{~ms}$ requirement for real-time processing at 30 frames per second. For an image of size $512 \times 512$ the processing time would be approximately four times greater, still assuming an $8 \times 8$ block size. This time could be reduced by a factor of two by using two arrays of thirty-two DCT chips and doubling the throughput rate of the mask processing components. This latter modification is not unreasonable considering the computational simplicity of the operations involved in the mask processing. 
While the circuitry required to implement the POCS transmitter is complex, the receiver is quite simple. All that is required is to compute the inverse cosine transform on the received coefficients and set all pixels outside the mask region to zero. Such imbalance between the complexity of the transmitter and the receiver is desirable in a teleconferencing system with multiple receivers. A block diagram of the receiver is presented in figure 2.

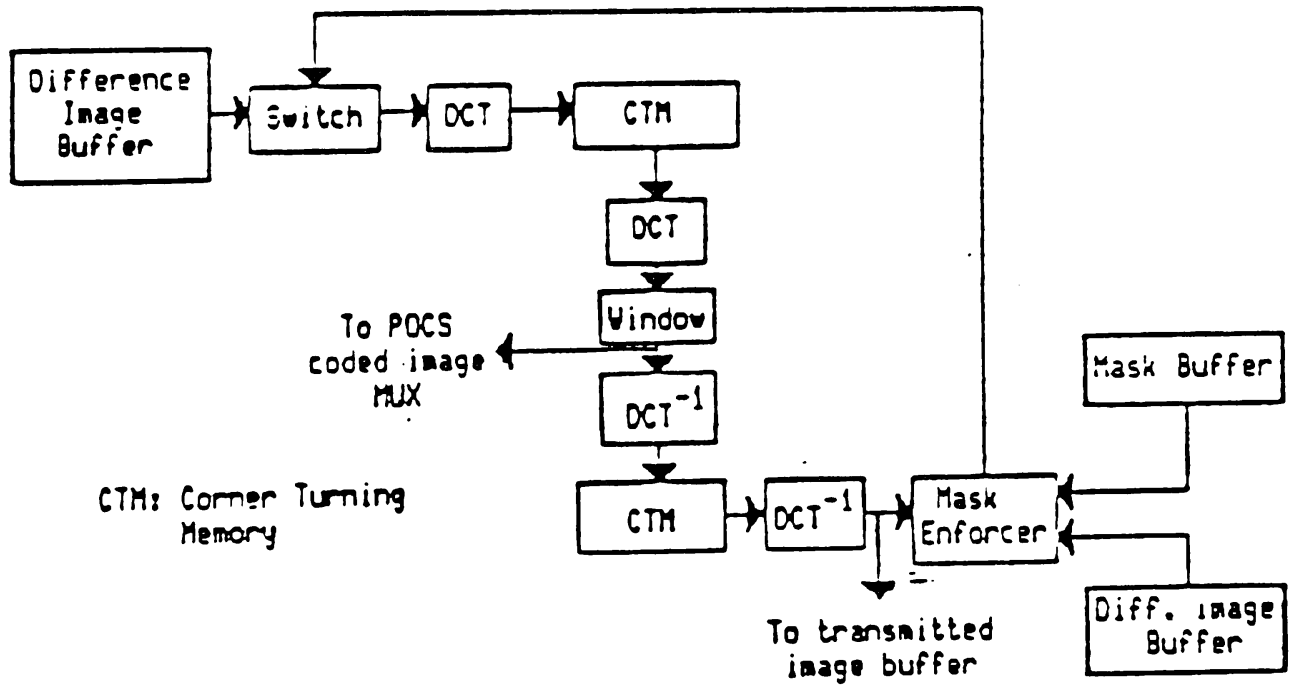

DCT COCER

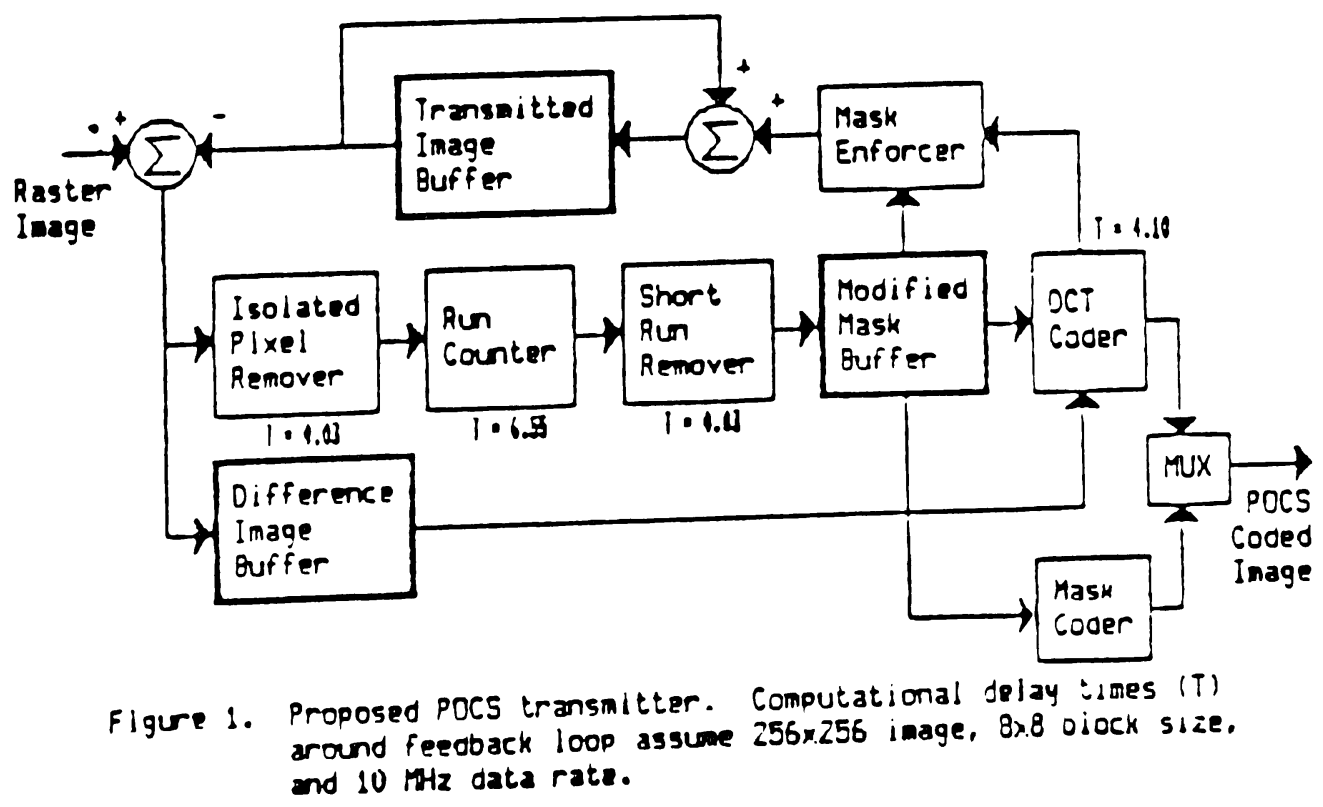




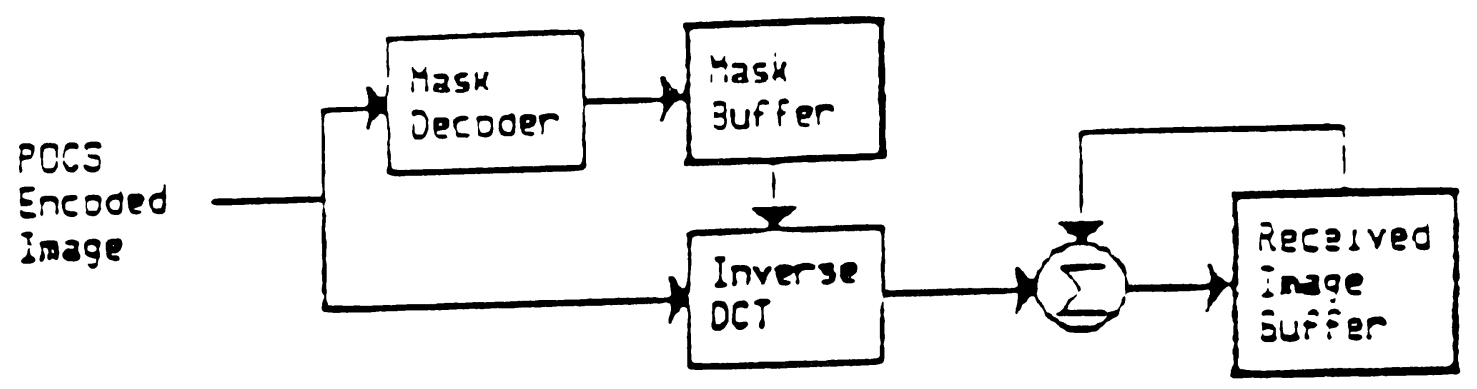

Figure 2 - POCS receiver.

It should be noted that the POCS codec will suffer from cumulative error in the reconstruction of the image from a sequence of difference images. This limitation can be easily overcome by gradually refreshing the picture as described in $\$$. sup $13 \$$

\section{Conclusions}

The use of the POCS theory in image sequence coding is in its early days. The possibility of employing various kinds of information in encoding images through the POCS method is promising. The application studied in this paper demonstrates the feasibility of a real-time POCS based codec for video teleconferencing applications.

\section{References}

[1] W. K. Pratt, Digital Image Processing, John Wiley \& Sons, New York, 1978.

[2] L. Gubin, B. Poljak, E. Raik, "The Method of Projections for Finding the Common Point of Convex Sets," USSR Computational Mathematics and Mathematical Physics, vol. 7, no. 6, pp. 1-24, 1967.

[3] H. J. Trussell, M. R. Civanlar, "The Feasible Solution in Signal Restoration," IEEE Transactions on Acoustics, Speech, and Signal Processing, vol. ASSP-32, no. 2, pp. 201212, April 1984. 
[4] W. Cheney, A. A. Goldstein, "Proximity Maps for Convex Sets," Proc. Amer. Math. Soc. vol. 10, pp 448-450, 1959.

[5] M. Goldburg, R. J. Marks, "Signal Synthesis in the Presence of an Inconsistent Set of Constraints," IEEE Transaction on Circuits and Systems, vol. CAS-32, no. 7, pp. 647663, July 1985.

[6] P. Santago and S. A. Rajala, "New Technique for Combined Pixel and Frequency Domain Coding Image Sequency Coding," MILCOM 1985, October 1985, Boston.

[7] P. Santago, "A composite model and convex set coding technique for time varying images," Ph.D Dissertation, January 1986, NCSU.

[8] P. Santago, S. A. Rajala, "Technique for Video Compression by Projection onto Convex Sets," SPIE 28th Annual Int. Technical Symp. on Optics and Electro-Optics, August 1984 .

[9] F. W. Mounts, "A Video Encoding System with Conditional Picture Element Replenishment," The Bell System Technical Journal, vol. 48, no. 7, pp. 2545-2554, September 1969.

[10] H. G. Musmann, P. Pirsch, H.-J. Grallert, "Advances in Picture Coding," Proceedings of the IEEE, vol. 73, no. 4, pp. 523-548, April 1985.

[11] W. A. Pearlman, P. Jakatdar, "The Effectiveness and Efficiency of Hybrid Transform/DPCM Interframe Image Coding," IEEE Transactions on Communications, vol. COM-32, no. 7, pp. 832-838, July 1984.

[12] M. R. Civanlar and P. Santago, "An Improved Transform Coder Using Attributes of the Difference Pictures," Proceedings of ICASSP-85, March 1985, Tampa, Florida.

[13] J. C. Candy, M. A. Franke, B. G. Haskell, F. W. Mounts, "Transmitting the Television as Clusters of Frame-to-Frame Differences," The Bell Systems Technical Journal, vol. 50, no. 6, pp. 1889-1917, 1971.

[14] J. D. Eggerton, M. D. Srinath, "A Visually Weighted Quantization Scheme for Image Bandwidth Compression at Low Data Rates," IEEE Transactions on Communications, vol. COM-34, no. 8, pp. 840-847, August 1986.

[15] Y. Yasuda, Y. Yamazaki, T. Kamae, K. Kobayashi, "Advances in FAX," Proceedings of the IEEE, vol. 73, no. 4, pp. 706-730, April 1985.

[16] M. Vetterli, A. Lightenberg, "A Discrete Fourier-Cosine Chip," IEEE Journal on Selected Areas in Communications, vol. SAC-4, no. 1, pp. 49-61, January 1986. 